\title{
Birationally rigid Fano cyclic covers over a hypersurface containing a singular point
}

\author{
Dominic Foord ${ }^{1}$
}

Received: 12 April 2019 / Revised: 16 November 2019 / Accepted: 25 April 2020 / Published online: 21 July 2020 (c) The Author(s) 2020

\begin{abstract}
Following on from the paper (Pukhlikov in Proc Edinb Math Soc 62(1):221-239, 2019), we prove birational superrigidity for a general Fano cyclic cover of a hypersurface containing a single isolated singular point on the base hypersurface lying off the ramification divisor, where the multiplicity of the point can be close to the degree. In particular, such a variety is not rational.
\end{abstract}

Keywords Birational rigidity $\cdot$ Maximal singularity $\cdot$ Multiplicity $\cdot$ Hypertangent divisor $\cdot$ Complete intersection singularity $\cdot$ Cyclic cover

Mathematics Subject Classification 14E05 · 14E07

\section{Introduction}

\subsection{Statement of the main result}

The aim of this paper is to prove birational superrigidity for a general Fano cyclic cover of a hypersurface with an isolated singular point of index one where the singular point does not lie on the ramification divisor and whose multiplicity can be close to the degree-we use the terminology "high multiplicity" to distinguish from the case where we only consider quadratic singularities with bounded rank. Let $M \geqslant 6$, and let $G=G_{m} \subset \mathbb{P}^{M+1}=\mathbb{P}$ be a hypersurface of degree $m$ containing a single isolated singular point $o^{\prime}$ with multiplicity $\mu$, where $\mu<M-4$. We then let

$$
\sigma: F \rightarrow G
$$

$凶$ Dominic Foord

Dominic.Foord@liverpool.ac.uk

1 Department of Mathematical Sciences, University of Liverpool, Mathematical Sciences Building, Liverpool L69 7ZL, UK 
be a $K: 1$ cyclic cover branched over a divisor $W \cap G$ where $W=W_{\mathrm{Kl}} \subset \mathbb{P}$ is a hypersurface of degree $\mathrm{Kl}$ and $o^{\prime} \notin W$. Introducing a new coordinate $u$ of weight $l$, we can realise $F$ as a complete intersection of type $m \cdot \mathrm{Kl}$ in the weighted projective space

$$
\mathbb{P}^{*}=\mathbb{P}(\underbrace{1, \ldots, 1}_{M+2}, l) .
$$

Namely, $F$ is given by the system of equations

$$
f\left(x_{0}, \ldots, x_{M+1}\right)=0, \quad u^{K}=g\left(x_{0}, \ldots, x_{M+1}\right)
$$

where $f$ and $g$ are homogeneous polynomials of degrees $m$ and $\mathrm{Kl}$ respectively. We further require that the polynomials $f$ and $g$ satisfy some regularity conditions at every point $p \in F$, stated in Sect. 2. Since $o^{\prime}$ is a singular point and $M \geqslant 6$, the variety remains factorial by [1]. Therefore, by [4], if the integers $m, l$ and $K$ satisfy the relation $m+(K-1) l=M+1$, then $F$ is a primitive Fano variety of dimension $M$, that is, Pic $F=\mathbb{Z} K_{F}$ and $\left(-K_{F}\right)$ is ample.

We also assume that

$$
(\mathrm{Kl})^{2}-5 \mathrm{~K} 1+10 \geqslant 2 m .
$$

The proof that the codimension of the subspace of the defining parameter space where the regularity conditions fail is positive requires this inequality.

The result of this paper is then:

Theorem 1.1 A general variety $F$ of the type described above is birationally superrigid. In particular, $F$ admits no non-trivial structures of a rationally connected fibration, any birational map $F \rightarrow F^{\#}$ onto a Fano variety with $\mathbb{Q}$-factorial terminal singularities and $\mathrm{rk} \operatorname{Pic} F^{\#}=1$ is an isomorphism and the groups of birational and biregular self-maps coincide:

$$
\text { Bir } F=\text { Aut } F \text {. }
$$

\subsection{Method of the proof}

We prove birational superrigidity of a given variety $F$ using the method of maximal singularities together with the technique of hypertangent divisors. We begin by letting $\Sigma$ be a mobile linear system on $F$ with a maximal singularity $E$ satisfying the NoetherFano inequality, i.e., we suppose $F$ is not birationally superrigid. We can easily exclude cases where the centre $B$ of $E$ has codimension less than $M$, and so we reduce to the case of a point. The case where the point is non-singular has already been covered in the paper [9] and so we must consider the case where it is singular. At this point, we can apply the generalised $4 n^{2}$ inequality to finish the proof. 
We then finally need to check whether the set of polynomials which fail the regularity conditions is of positive codimension in the parameter space of defining polynomials.

\subsection{Historical remarks}

The notion of birational superrigidity goes back to the paper [5] where Iskovskikh and Manin proved the non-rationality of the smooth quartic threefold using the method of maximal singularities. This method was refined in the ' 90 s and was used successfully for many classes of index 1 Fano varieties. Related to our paper, we have the following: double covers were studied in the paper [6], followed by the study of iterated double covers in the paper [7]. Birational superrigidity of singular hypersurfaces were considered in the paper [8]. Later on the superrigidity of a double cover over a cubic or a quartic and a triple cover with at worst ordinary double points was proved in the papers [3] and [2] respectively. Most recently, we have the paper [12], on whose result the main theorem of this paper is based.

\section{The regularity conditions}

We describe the conditions for generality of the variety $F$. Since $F$ is determined by two polynomials $f$ and $g$ of degrees $m$ and $\mathrm{Kl}$ respectively, we can view $F$ as a point $\underline{f}$ in the parameter space $\mathcal{F}$

$$
\underline{f} \in \mathcal{F} \subset \mathbb{P}\left(H^{0}\left(\mathbb{P}, \mathcal{O}_{\mathbb{P}}(m)\right)\right) \times \mathbb{P}\left(H^{0}\left(\mathbb{P}, \mathcal{O}_{\mathbb{P}}(\mathrm{Kl})\right)\right)
$$

under the following conditions:

- For a pair of polynomials $(f, g)=f \in \mathcal{F}$, the corresponding Fano cyclic cover $F=\mathbb{V}(f, g) \subset \mathbb{P}^{*}$ is irreducible and reduced.

- Deconstructing the local equation for $f$ at the point $o^{\prime}$ (here we view $f$ as defining a variety on the base) into homogeneous components, the initial $\mu-1$ components all vanish, that is to say locally

$$
f=q_{\mu}+q_{\mu+1}+\cdots+q_{m}
$$

where $q_{i}$ is the $i$ th homogeneous component of $f$ and $q_{\mu}$ is not identically zero, whilst at every other point $o$, the equation is locally given in homogeneous components as

$$
f=q_{1}^{\prime}+q_{2}^{\prime}+\cdots+q_{m}^{\prime}
$$

where again $q_{1}$ is not identically zero.

- The variety defined by the vanishing of the polynomial $u^{K}=g$ is non-singular.

This set $\mathcal{F}$ makes a natural parameter space for the Fano cyclic covers under study, and is clearly open in the projective subspace of the total parameter space. 
We also need a condition on the blow-up of the singular point $o^{\prime} \in G$ on the base hypersurface:

(R0.1) Condition on the singularity on the base. Let $\varphi_{\mathbb{P}}: \mathbb{P}^{+} \rightarrow \mathbb{P}$ be the blow-up of the point $o^{\prime}$ on $G, E_{\mathbb{P}}=\varphi_{\mathbb{P}}^{-1}\left(o^{\prime}\right) \cong \mathbb{P}^{M}$ the exceptional divisor, $G^{+} \subset \mathbb{P}^{+}$the strict transform of the hypersurface $G$, so that $\varphi: G^{+} \rightarrow G$ is the blow-up of the point $o^{\prime}$ on $G$ and $E=G^{+} \cap E_{\mathbb{P}}$ is the exceptional divisor. We require that the subvariety

$$
E \subset E_{\mathbb{P}} \cong \mathbb{P}^{M}
$$

is a non-singular hypersurface in its linear span, i.e.,

$$
\langle E\rangle \cong \mathbb{P}^{M}
$$

In other words, supposing $\left(z_{0}, z_{1}, \ldots, z_{M+1}\right)$ is a set of affine coordinates at the point $o^{\prime}$, with the local equation again decomposed into homogeneous coordinates as

$$
f=q_{\mu}+q_{\mu+1}+\cdots+q_{m}
$$

then we ask that $\left(z_{0}: \ldots: z_{M+1}\right)$ forms a set of affine coordinates on $E_{\mathbb{P}}$ and the hypersurface $E$ is given by the equation $\left.q_{\mu}\right|_{E_{\mathbb{P}}}=0$. This condition is to ensure that at singular points on the cover in question, we can apply the generalised $4 n^{2}$-inequality, Theorem 3.4.

Now let $f=(f, g) \in \mathcal{F}$ be a defining pair for a Fano cyclic cover $F, p \in F$ an arbitrary point, and let $p^{\prime}=\sigma(p)$. We choose a system of affine coordinates $z_{1}, \ldots, z_{M+1}$ with the origin at the point $p^{\prime}$. Without loss of generality we can assume that $z_{1}=x_{i} / x_{0}$. We set $y=u / x_{0}^{l}$.

Then the standard affine set

$$
\mathbb{A}=\mathbb{A}_{\left(z_{1}, \ldots, z_{M+1}, y\right)}^{M+2}
$$

is a chart for $\mathbb{P}(1, \ldots, 1, l)$. Abusing notation, we use the same symbols corresponding to the homogeneous polynomials $f$ and $g$, namely $f=q_{1}^{\prime}+q_{2}^{\prime}+\cdots+q_{m}^{\prime}$ at smooth points of the intersection, $f=q_{\mu}+q_{\mu+1}+\cdots+q_{m}$ at singular points, and $g=w_{0}+w_{1}+\cdots+w_{\mathrm{K} 1}$, where the polynomials $q_{i}^{\prime}, q_{j}$ and $w_{k}$ are homogeneous components of degree $i, j$ and $k$ respectively in the variables $z_{*}$, so that in the affine chart $\mathbb{A}$, the variety $F$ is given by the pair of equations $f=0, y^{K}=g$, replacing our original system. If the point $p \in F$ does not lie on the ramification divisor, then we assume that $w_{0}=1$. If it does, the point $p^{\prime} \in G$ is non-singular, and so without loss of generality, we assume that $q_{1} \equiv z_{M+1}$.

We now formulate the regularity condition for any point $o \in F$.

(R1.1) The regularity condition for a point $p$ outside the ramification divisor. Let the singularities of $F$ be given by the set

$$
\text { Sing } F=\left\{o_{1}^{\prime}, o_{2}^{\prime}, \ldots, o_{K}^{\prime}\right\}
$$


where the points $o_{1}^{\prime}, \ldots, o_{K}^{\prime}$ are the $K$ points in the preimage of the singular point $o^{\prime} \in G$. Let $p$ be one of the points $o_{i}^{\prime} \in \operatorname{Sing} F$. We assume locally $w_{0}=1$ and we may also assume that $y(p)=1$. Set

$$
\begin{aligned}
g^{1 / K}=\left(1+w_{1}+\cdots+w_{\mathrm{Kl}}\right)^{1 / K} & =1+\sum_{i=1}^{\infty} \gamma_{i}\left(w_{1}+\cdots+w_{\mathrm{Kl}}\right)^{i} \\
& =1+\sum_{i=1}^{\infty} \Phi_{i}\left(w_{1}, \ldots, w_{\mathrm{K} l}\right)
\end{aligned}
$$

where $\gamma_{i} \in \mathbb{Q}$ are the coefficients in the Taylor expansion of $(1+s)^{1 / K}$ at zero and $\Phi_{i}\left(w_{1}\left(z_{*}\right), \ldots, w_{\mathrm{Kl}}\left(z_{*}\right)\right)$ are homogeneous polynomials of degree $i \geqslant 1$ in the variables $z_{*}$. It is easy to see that for $i \in\{1, \ldots, \mathrm{Kl}\}$ we get

$$
\Phi_{i}\left(w_{*}\left(z_{*}\right)\right)=\frac{1}{K} w_{i}+\Phi_{i}^{\#}\left(w_{1}, \ldots, w_{i-1}\right)
$$

for some polynomials $\Phi_{i}^{\#}$, only depending on the polynomials $\left(w_{1}, \ldots, w_{i-1}\right)$. In these notations we formulate the regularity condition in the following way: We say a sequence satisfies the regularity condition (R1.1) if the set of polynomials

$$
\left\{q_{\mu}, \ldots, q_{m}, \Phi_{l+1}\left(w_{*}\left(z_{*}\right)\right), \ldots, \Phi_{v}\left(w_{*}\left(z_{*}\right)\right)\right\}
$$

forms a regular sequence in $\mathcal{O}_{p, \mathbb{C}^{M+1}}$, where

$$
v= \begin{cases}\frac{\mathrm{Kl}}{2}+1 & \text { if } \mathrm{Kl} \text { is even } \\ \frac{\mathrm{Kl}+1}{2} & \text { if } \mathrm{Kl} \text { is odd }\end{cases}
$$

In other words, the set of homogeneous equations

$$
\left\{q_{i}=0, \Phi_{j}=0 \mid i=\mu, \ldots, m, j=l+1, \ldots, v\right\}
$$

defines a closed set of codimension $v+m-\mu-l+1$ in $\mathbb{C}^{M+1}$.

Considering now a non-singular point $p \neq o_{i}^{\prime}, i \in\{1, \ldots, K\}$, lying off the ramification divisor, the conditions are identical to those in the paper [9]. Let $u_{1}, \ldots, u_{M+1}$ be a system of affine coordinates with the origin at $p$. We perform the same decomposition as before, finishing with local equations

$$
\left\{q_{1}^{\prime}, \ldots, q_{m}^{\prime}, \Phi_{1}^{\prime}\left(w_{*}^{\prime}\left(u_{*}\right)\right), \Phi_{2}^{\prime}\left(w_{*}^{\prime}\left(u_{*}\right)\right), \ldots\right\}
$$

at the point $p$. We then require the regularity of the sequence

$$
\left\{q_{1}^{\prime}, \ldots, q_{m}^{\prime}, \Phi_{l+1}^{\prime}\left(w_{*}^{\prime}\left(u_{*}\right)\right), \ldots, \Phi_{\mathrm{K} 1-1}^{\prime}\left(w_{*}^{\prime}\left(u_{*}\right)\right)\right\}
$$


if $m \leqslant \mathrm{Kl}$, whilst if $m>\mathrm{K} 1$, we require the regularity of the sequence

$$
\left\{q_{1}^{\prime}, \ldots, q_{m-1}^{\prime}, \Phi_{l+1}^{\prime}\left(w_{*}^{\prime}\left(u_{*}\right)\right), \ldots, \Phi_{\mathrm{K} 1}^{\prime}\left(w_{*}^{\prime}\left(u_{*}\right)\right)\right\}
$$

Note that we also call this condition (R1.1).

(R1.2) The regularity condition for a point $p$ on the ramification divisor. Here $w_{0}=0$. We require that the set of polynomials

$$
\left\{q_{1}^{\prime \prime}, \ldots, q_{m}^{\prime \prime}, w_{1}^{\prime \prime}, \ldots, w_{K}^{\prime \prime}\right\}
$$

forms a regular sequence in $\mathcal{O}_{p}, \mathbb{C}^{M+1}$, where $q_{i}^{\prime \prime}$ and $w_{i}^{\prime \prime}$ are the local defining equations at the point $p$.

Definition 2.1 A Fano cyclic cover defined by $f \in \mathcal{F}$ is said to be regular, if every point $p$ in the corresponding variety $F$ satisfies the regularity conditions, namely the conditions (R1.1) and (R1.2), and the singularity on the base satisfies the condition (R0.1).

We denote the set of regular cyclic covers by the symbol $\mathcal{F}_{\text {reg. }}$ This is clearly open in $\mathcal{F}$.

Theorem 2.2 The set $\mathcal{F}_{\text {reg }}$ is non-empty and the following inequality holds:

$$
\operatorname{codim}\left(\mathcal{F} \backslash \mathcal{F}_{\text {reg }} \subset \mathcal{F}\right) \geqslant 2 \text {. }
$$

We postpone the proof of this for now, and first of all show the proof of Theorem 1.1.

\section{Proof of Theorem 1.1}

Assuming Proposition 2.2, we can now prove Theorem 1.1; we show that any regular cyclic cover $F$ corresponding to a point $\underline{f} \in \mathcal{F}_{\text {reg }}$ is birationally superrigid.

\subsection{The maximal singularity}

We fix a pair of polynomials $f=(f, g) \in \mathcal{F}_{\text {reg. }}$. Let $F=\mathbb{V}(f, g)$ be the corresponding cyclic cover. We repeat that by our choices of $m, \mu, K$ and $l$, we have that

$$
\operatorname{Pic} F=\mathbb{Z} H, \quad K_{F}=-H,
$$

where $H$ is the $\sigma$-pull-back of a hyperplane section of $G$.

Assume that $F$ is not birationally superrigid. By what was said before, this implies that on $F$ there is a mobile linear system $\Sigma \subset|n H|, n \geqslant 1$, with a maximal singularity $E$ : for some non-singular projective variety $\widetilde{F}$ with a birational morphism $\varphi: \widetilde{F} \rightarrow$ $F$ there exists a $\varphi$-exceptional prime divisor $E \subset \widetilde{F}$ satisfying the Noether-Fano inequality

$$
\operatorname{ord}_{E} \Sigma>n a(E)
$$


Let $B=\varphi(E) \subset F$ be the centre of the divisor $E$ on $F$. This is an irreducible subvariety satisfying the inequality mult ${ }_{B} \Sigma>n$. By the Lefschetz theorem for numerical Chow groups of algebraic cycles on $V$, we have the equality $A^{2} V=\mathbb{Z} H^{2}$. We can now exclude the simplest case where $\operatorname{codim}(B \subset F)=2$.

If $\operatorname{codim}(B \subset F)=2$, then we begin by intersecting with a general six dimensional linear subvariety $V \subset \mathbb{P}^{*}$, so that we have a five dimensional smooth variety $F_{V}=$ $F \cap V$. Further set $H_{V}=H \cap V$. Then by the Lefschetz theorem,

$$
\operatorname{Pic} F_{V}=\mathbb{Z} H_{V}, \quad A^{2} F_{V}=\mathbb{Z} H_{V}^{2}
$$

If we also set $B_{V}=B \cap V$, then $B_{V} \sim m H_{V}^{2}$ for some $m \geqslant 1$. Consider the selfintersection $Z=\left(D_{1} \circ D_{2}\right)$ of the linear system $\Sigma_{V}=\Sigma \cap V$. Clearly $Z \sim n^{2} H_{V}^{2}$. On the other hand, $Z=\gamma B_{V}+Z_{1}$ where $\gamma>n^{2}$ and $Z_{1}$ is an effective cycle of codimension 2 that does not contain $B_{V}$ as a component (here we are using the Noether-Fano inequality together with, for example, [10, Chapter 2, Lemma 2.2]). Taking the classes of the cycles in $A^{2} F_{V}$ then yields the inequality $n^{2} \geqslant \gamma m>m n^{2}$. This is the required contradiction.

If $\operatorname{codim}(B \subset F) \geqslant 3$ and $B \not \subset$ Sing $F$, then the inequality mult ${ }_{B} Z>4 n^{2}$ holds. This is the classical $4 n^{2}$-inequality going back to [5] (see [10, Chapter 2] for a modern exposition). At this point, we use the arguments of [10, Chapter 3, Section 2, Theorem $2.1]$ to cover this case. We can do this because it relies only on the regularity conditions at non-singular points.

We are therefore left with the only option: $B$ is a singular point lying off the ramification divisor, specifically $B=o \in\left\{o_{1}^{\prime}, \ldots, o_{K}^{\prime}\right\}$. To do this, we use the method of hypertangent linear systems.

\subsection{Hypertangent linear systems}

Definition 3.1 Let $X$ be a variety, and let $\pi: X^{+} \rightarrow X$ be the blow-up of an arbitrary point $o \in X$. Assume that the exceptional divisor $E=\pi^{-1}(o)$ is reduced and irreducible. An effective divisor on $X$ is said to be hypertangent to $X$ (with respect to a point $o$ ) if the strict transform of the divisor $D$, denoted $D^{+}$, belongs to the linear system $|k H-l E|$, where $l \geqslant k+1$. The number $\beta(D)=l / k$ is then called the slope of the divisor.

Following from this definition, returning to our original cover

$$
\sigma: F \rightarrow G
$$

set

$$
D_{i}=\sigma^{*} \overline{\left\{\left.\left(q_{\mu}+\cdots+q_{i}\right)\right|_{G}=0\right\}}
$$


where $i=\mu, \ldots, m-1$ and where we are taking the closure in $\mathbb{P}^{*}$. Similarly, let

$$
L_{j}=\overline{\left\{y-1-\left.\sum_{i=1}^{j} \Phi_{i}\left(w_{1}, \ldots, w_{j}\right)\right|_{F}=0\right\}}
$$

where $j=l, \ldots, v-1$. These sets are clearly both of hypertangent divisors with multiplicities at the point $o$ of

$$
\operatorname{mult}_{o} D_{i}=i+1, \quad \operatorname{mult}_{o} L_{j}=j+1 \text {, }
$$

and hence with slopes

$$
\beta\left(D_{i}\right)=\frac{i+1}{i}, \quad \beta\left(L_{j}\right)=\frac{j+1}{j}
$$

respectively. To see this note that $\left.\left(q_{\mu}+\cdots+q_{i}\right)\right|_{G}=\left.\left(-q_{i+1}-\cdots-q_{m}\right)\right|_{G}$ and similarly for the divisors $L_{j}$. Define the set

$$
\mathcal{D}=\left\{D_{i} \mid i=\mu, \ldots, m-1\right\} \cup\left\{L_{j} \mid j=l \ldots, v-1\right\} .
$$

to be the collection of these hypertangent divisors. Let

$$
N=\# \mathcal{D}=m-\mu+v-l
$$

Further let $H$ as before. Note that as $H$ is ample, $|H|$ is free and hence defines a finite morphism

$$
\alpha=\alpha_{|H|}: X \rightarrow \mathbb{P}^{\operatorname{dim}|H|} .
$$

Definition 3.2 Let $\pi: X^{+} \rightarrow X$ be the blow-up of $X$ at the point $o$. A non-empty linear system $\Sigma$ on $X$ is said to be hypertangent (with respect to the point $o$ ) if $\Sigma^{+} \subset|k H-l E|$, where $E$ is the exceptional divisor, $l \geqslant k+1$, and $\Sigma^{+}$is the strict transform of the system $\Sigma$ on $X^{+}$. The number $\beta(\Sigma)=l / k>1$ is called the slope of the system $\Sigma$.

The point is that our set of hypertangent divisors $\mathcal{D}$ generates a hypertangent linear system $\Sigma_{k}=\Sigma_{k}(\mathcal{D})$ in the following way: for each $D \in \mathcal{D}$, define $k_{D}$ and $l_{D}$ to be the coefficients in the expression of the strict transform $D^{+} \in\left|k_{D} H-l_{D} E\right|$. Let

$$
f_{D} \in H^{0}\left(X, \mathcal{O}_{X}\left(k_{D} H\right)\right)
$$

be a section defining the divisor $D$. Set

$$
\Sigma_{k}=\left|\sum_{k_{D} \leqslant k} f_{D} s_{D}=0\right|
$$


where the summation is taken over all hypertangent divisors $D \in \mathcal{D}$ such that $k_{D} \leqslant k$, and $s_{D} \in \alpha^{*} H^{0}\left(\mathbb{P}^{\operatorname{dim}|H|}, \mathcal{O}\left(k-k_{D}\right)\right)$ is the pull-back of an arbitrary polynomial of degree $k-k_{D}$ with a zero of order $k-k_{D}$ at the point $\alpha(o)$. Clearly

$$
\beta\left(\Sigma_{k}\right) \geqslant \min _{\substack{D \in \mathcal{D} \\ k_{d} \leqslant k}}\left\{\frac{k+l_{D}-k_{D}}{k}\right\} .
$$

In fact, in our case, for every $D \in \mathcal{D}$ we have that $l_{D}=k_{D}+1$, so that all the slopes are of the form

$$
\beta\left(\Sigma_{k}\right) \geqslant \frac{k+1}{k}
$$

Since $\alpha$ is finite, we get the equality

$$
\operatorname{codim}_{o} \operatorname{Bs} \Sigma_{k}=\#\left\{D \in \mathcal{D} \mid k_{D} \leqslant k\right\}
$$

where Bs denotes the base locus of the linear system in question. From this equality, we see that the integer-valued function $\operatorname{codim}_{o} \mathrm{Bs} \Sigma_{k}$ is increasing when $k=k_{D}$ for some $D \in \mathcal{D}$, and only for those values.

Define the ordering function $\chi:\{1, \ldots, N\} \rightarrow \mathcal{K}=\left\{k_{D} \mid D \in \mathcal{D}\right\}$ by the relation

$$
\#\left\{D \in \mathcal{D} \mid k_{D}<\chi(i)\right\}<i \leqslant \#\left\{D \in \mathcal{D} \mid k_{D} \leqslant \chi(i)\right\} .
$$

For example,

$$
\chi(1)=\min \left\{k_{D} \mid D \in \mathcal{D}\right\}, \quad \chi(N)=\max \left\{k_{D} \mid D \in \mathcal{D}\right\} .
$$

By construction, we finally obtain

$$
\operatorname{codim}_{o} \operatorname{Bs} \Sigma_{\chi(i)} \geqslant i
$$

From this, pick a general set of hypertangent divisors

$$
\mathbb{D}=\left(D_{1}, \ldots, D_{N}\right) \in \prod_{i=1}^{N} \Sigma_{\chi(i)}
$$

and an arbitrary subvariety $Y$ of codimension $d$ containing the point $o$; we get $Y \not \subset$ $\operatorname{Supp}\left(D_{i}\right)$ for $i \geqslant d+1$. In particular, let us take $Y$ to be an irreducible component of the self-intersection $Z$ with the highest ratio mult $t_{o} / \mathrm{deg}$ of the multiplicity at the point $o$ to the degree $d$; this is clearly bounded above by 1 . We can now construct in the usual way (see [10, Chapter 3]) a sequence of irreducible subvarieties $Y_{2}=Y, Y_{3}, \ldots, Y_{N}$ satisfying the following properties: 
- $\operatorname{codim}\left(Y_{i} \subset F\right)=i$,

- $Y_{i} \not \subset D_{\chi(i+1)}$, so that $\left(Y_{i} \circ D_{\chi(i+1)}\right)$ is an effective cycle on $V$ and $Y_{i+1}$ is one of its irreducible components,

- $Y_{i+1}$ is an irreducible component of the algebraic cycle of the scheme-theoretic intersection $\left(Y_{i} \circ D_{\chi(i+1)}\right)$ with the maximal possible value of mult $Y_{i+1} / \operatorname{deg} Y_{i+1}$.

In particular, the inequality

$$
\frac{\operatorname{mult}_{o} Y_{i+1}}{\operatorname{deg} Y_{i+1}} \geqslant \beta\left(\Sigma_{i+1}\right) \frac{\operatorname{mult}_{o} Y_{i}}{\operatorname{deg} Y_{i}}
$$

holds, again using [10, Chapter 2, Lemma 2.2], which immediately implies the following proposition.

Proposition 3.3 The following inequality holds:

$$
\frac{\operatorname{mult}_{o} Y}{\operatorname{deg} Y} \leqslant\left(\prod_{i=1}^{N-2} \beta\left(\Sigma_{d+i}\right)\right)^{-1} .
$$

We now use the following theorem, a generalisation of the $4 n^{2}$-inequality proved by Pukhlikov in [11].

Theorem 3.4 Let $X$ be a degree $m$ complete intersection with a generic singularity $o$ (i.e., $X$ satisfies the condition (R0.1)) where mult $_{o} X=\mu \leqslant m$ and

$$
\operatorname{dim} X=M \geqslant \mu+4 \text {. }
$$

Let $\Sigma$ be a mobile linear system on $X$. Assume that for some positive $n \in \mathbb{Q}$ the pair $\left(X, \frac{1}{n} \Sigma\right)$ is not canonical at the point o but canonical outside this point. Then the self-intersection $Z=\left(D_{1} \circ D_{2}\right)$ of the system $\Sigma$ satisfies the inequality

$$
\text { mult }_{o} Z>4 \mu n^{2} \text {. }
$$

Returning to our variety $Y \subset F$, we apply Theorem 3.4 to get the following inequality, supposing that $F$ is birationally superrigid:

$$
\frac{\operatorname{mult}_{o} Y}{\operatorname{deg} Y} \leqslant \frac{4 \mu}{m K},
$$

which holds at every singular point $o \in F$, where the degree is defined by the formula: $\operatorname{deg} Y=\left(Y \cdot H^{M-2}\right)$.

Theorem 1.1 then follows if this holds true; to see this, note that $F$ being birationally superrigid would imply that $\operatorname{deg} Z=m K n^{2}$ and mult ${ }_{o} Z>4 \mu n^{2}$. This contradicts the above. 
If we now apply Proposition 3.3 to the subvariety $Y$, we can hence deduce the following:

$$
\begin{aligned}
\frac{\operatorname{mult}_{o} Y}{\operatorname{deg} Y} & \leqslant\left(\frac{\mu}{\mu+1} \cdot \frac{\mu+1}{\mu+2} \cdot \prod_{i=\mu}^{m-1} \frac{i+1}{i} \cdot \prod_{j=l}^{\nu-1} \frac{j+1}{j}\right)^{-1} \\
& =\frac{\mu+2}{\mu} \cdot \frac{\mu \cdot l}{m v}<\frac{(\mu+2) \cdot 2}{m K} \leqslant \frac{4 \mu}{m K},
\end{aligned}
$$

which proves the inequality (3).

\section{Proof of Theorem 2.2}

Let us prove Theorem 2.2. This follows from the following: $\mathcal{F}$ is non-empty-we show this in the proof of Theorem 5.1. Now let $p \in \mathbb{P}^{*}$ be an arbitrary fixed point, and let $p^{\prime}=\sigma(p)$. Consider the set $\mathcal{F}(p)=\left\{\underline{f} \in \mathcal{F} \mid G \ni p^{\prime}\right\} \subset \mathcal{F}$. Since the cover $\sigma$ is cyclic, either all the points $\sigma^{-1}\left(p^{\prime}\right)$ satisfy the regularity conditions, or none of them do. Set

$$
\mathcal{F}_{\text {reg }}(p) \subset \mathcal{F}(p)
$$

to be the set of coverings such that each point $p^{\prime} \in \sigma^{-1}(p)$ satisfies the regularity conditions defined previously.

Proposition 4.1 The following inequality holds:

$$
\operatorname{codim}_{\mathcal{F}(p)}\left(\mathcal{F}(p) \backslash \mathcal{F}_{\text {reg }}(p) \subset \mathcal{F}(p)\right) \geqslant M+2
$$

Since $p \in \mathbb{P}^{M+1}$ is an arbitrary point, and $\mathcal{F}(p) \subset \mathcal{F}$ is a divisor, we can use the same argument as in the case of a Fano complete intersection (see [10, Chapter 3, Section 3, 3.2]) to complete the proof of Proposition 2.2.

First of all, the case where $p$ is non-singular has been covered in [9, Proposition 5.1]. Therefore, we assume that $p$ is singular (and hence has multiplicity $\mu$ ).

We need the following lemma. We construct a sequence of polynomials $\Phi_{i}^{+}\left(w_{1}\right.$, $\left.\ldots, w_{l}\right)$, setting

$$
\Phi_{l+1}^{+}=\Phi_{l+1}^{\#}
$$

and then subsequently

$$
\Phi_{i}^{+}=\Phi_{i}^{\#}\left(w_{1}, \ldots, w_{l},-K \Phi_{l+1}^{+}, \ldots,-K \Phi_{i-1}^{+}\right)
$$

for $i \geqslant l+2$. 
Lemma 4.2 The sequence (2) is regular if and only if the set of polynomials

$$
\left\{q_{\mu}, \ldots, q_{m}, w_{l+1}+K \Phi_{l+1}^{+}, \ldots, w_{v}+K \Phi_{v}^{+}\right\}
$$

forms a regular sequence.

Proof The sets of zeros for both sequences are the same: this can be shown by induction using the equality

$$
\left.\left.\Phi_{i}\right|_{B} \equiv 0 \Longleftrightarrow w_{i}\right|_{B} \equiv-K \Phi_{i}^{\#}
$$

for an arbitrary closed irreducible set $B$.

Note that the sequence (4) has the polynomials $w_{i}\left(z_{*}\right)$ shifted by polynomials $\Phi_{i}^{+}$ which depend only on $w_{1}, \ldots, w_{l}$. The set of polynomials $w_{i}, i \in\{1, \ldots, l\}$, can be assumed to be a fixed set of general position. Therefore in (4) each of the homogeneous polynomials $w_{i}, i \in\{l+1, v\}$, is shifted by a fixed homogeneous polynomial of degree $i$.

Let $\Pi$ be the space of polynomials $q_{\mu}, \ldots, q_{m}, w_{1}, \ldots, w_{\nu}$. Consider an irreducible component $X \subset \Pi$ corresponding to non-regular sequences (2) or (4). For a fixed set of homogeneous polynomials $u_{1}, \ldots, u_{l}$, where $\operatorname{deg} u_{i}=i$, let $\Pi\left(u_{1}, \ldots, u_{l}\right)=\left\{w_{i}=u_{i} \mid i=1, \ldots, l\right\} \subset \Pi$ be the corresponding affine subspace with fixed polynomials $w_{1}, \ldots, w_{l}$, and set $X\left(u_{1}, \ldots, u_{l}\right)=X \cap \Pi\left(u_{1}, \ldots, u_{l}\right)$. $\Pi\left(u_{1}, \ldots, u_{l}\right)$ is identified with the space of polynomials $q_{\mu}, \ldots, q_{m}, w_{l+1}, \ldots, w_{\nu}$, which we denote by $\Pi^{+}$. Thus we consider $X\left(u_{1}, \ldots, u_{l}\right)$ to be embedded in the linear space $\Pi^{+}$. For a general tuple $\left(u_{1}, \ldots, u_{l}\right)$ we have

$$
\operatorname{codim}_{\Pi} X=\operatorname{codim}_{\Pi^{+}} X\left(u_{1}, \ldots, u_{l}\right)
$$

Lemma $4.3 X(0, \ldots, 0) \neq \varnothing$.

Proof $\Phi_{i}, \Phi_{i}^{\#}$ and $\Phi_{i}^{+}$are quasi-homogeneous in $w_{*}$, where wt $w_{i}=i$. Therefore, for $\lambda \neq 0$,

$$
\left(q_{\mu}, \ldots, q_{m}, \lambda^{l+1} w_{l+1}, \ldots, \lambda^{\nu} w_{\nu}\right) \in X\left(\lambda u_{1}, \lambda^{2} u_{2}, \ldots, \lambda^{l} u_{l}\right)
$$

if and only if

$$
\left(q_{\mu}, \ldots, q_{m}, w_{l+1}, \ldots, w_{v}\right) \in X\left(u_{1}, \ldots, u_{l}\right)
$$

Setting $\lambda=0$ gives us the statement, using closure of the component.

Remark 4.4 If we then notice that

$$
\operatorname{codim}_{\Pi} X \geqslant \operatorname{codim}_{\Pi^{+}} X(0, \ldots, 0),
$$

this allows us to calculate the codimension of the space where the regularity conditions fail, essentially by ignoring the polynomials $\Phi_{i}^{+}$, and so we only need to estimate the 
codimension of the closed set of non-regular sequences $q_{\mu}, \ldots, q_{m}, w_{l+1}, \ldots, w_{\nu}$, taking into account the effect that introducing the singularity will affect the proof of regularity of non-singular points in the same neighbourhood. In fact, we consider the larger set of non-regular sequences $q_{\mu}, \ldots, q_{m}, w_{l+1}, \ldots, w_{\mathrm{Kl}}$, as the bound is good enough for our purposes.

\section{Codimension estimate}

Begin first of all by noting that the codimension estimate is trivial at the point $o$, and follows from the usual argument for a codimension count in the smooth case. However, as we will see, it is possible the singular point will have an effect on the codimension of the set of non-regular sequences at nearby smooth points, so we have to check that the regularity conditions hold here as well.

Recall that we are working in the chart $\mathbb{A}$ where the $y$ coordinate is fixed to be equal to 1 , and by abuse of notation whenever we talk about the variety $F$, we are referring to its restriction to the chart $\left.\mathbb{A}\right|_{\{y=1\}}$. We let $\mathcal{P}_{d, M+1}$ stand for the linear space of homogeneous polynomials of degree $d$ in $M+1$ variables $\left(z_{*}\right)$. Set

$$
\mathcal{P}_{[a, b], M+1}=\prod_{l=a}^{b} \mathcal{P}_{l, M+1}
$$

to be the space of tuples of polynomials of the form $\left(q_{a}, q_{a+1}, \ldots, q_{b}\right)$, where $q_{d} \in$ $\mathcal{P}_{d, M+1}$. We then let

$$
\mathcal{P}=\mathcal{P}_{[\mu, m], M+1} \times \mathcal{P}_{[l+1, \mathrm{Kl}], M+1}
$$

to be the space of pairs $f$ of defining polynomials of the type discussed above. In the following, $F$ always refers to the corresponding variety. Note that for a general pair the condition (R0.1) is satisfied.

Let $o \in F$ be a singular point lying at the origin in $\mathbb{A}$ and let $p \in \mathbb{A}, p \notin$ $\left\{o_{1}, \ldots, o_{K}\right\}$, be an arbitrary point. We assume that $p$ has coordinates $(1,0, \ldots, 0)$. We let

$$
u_{*}=\left\{u_{1}=z_{1}-1, u_{2}=z_{2}, \ldots, u_{M+1}=z_{M+1}\right\}
$$

be a system of affine coordinates with origin at the point $p$. Set $q_{j}=q_{j, k}+z_{1} q_{j, k-1}+$ $\cdots+z_{1}^{k} q_{j, 0}$ and $w_{j}=w_{j, k}+z_{1} w_{j, k-1}+\cdots+z_{1}^{k} w_{j, 0}$ where $q_{j, k}$ and $w_{j, k}$ are homogeneous polynomials of degree $k \leqslant j$ in the variables $z_{2}, \ldots, z_{M+1}$. In the alternate coordinates $u_{*}$, we can see that the polynomial $q_{j}^{\prime}$ takes the form

$$
\sum_{\alpha=0}^{j} u_{1}^{j-\alpha}\left(\sum_{\delta=\max (\mu, j)}^{m}\left(\begin{array}{l}
\delta-\alpha \\
j-\alpha
\end{array}\right) q_{\delta, \alpha}\right) .
$$


Similarly, $w_{j}^{\prime}$ takes the form

$$
\sum_{\alpha=0}^{j} u_{1}^{j-\alpha}\left(\sum_{\delta=j}^{\mathrm{K} l}\left(\begin{array}{l}
\delta-\alpha \\
j-\alpha
\end{array}\right) w_{\delta, \alpha}\right) .
$$

Therefore, the change of coordinates $\left(z_{*}\right) \rightarrow\left(u_{*}\right)$ defines a linear map

$$
\tau: \mathcal{P} \rightarrow \mathcal{P}_{[0, m], M+1}\left(u_{*}\right) \times \mathcal{P}_{[0, \mathrm{Kl}], M+1}\left(u_{*}\right)
$$

Theorem 5.1 The set of pairs $\underline{f}$ in $\mathcal{P}$ such that

$$
\tau(\underline{f}) \in \mathcal{P}_{[1, m], M+1}\left(u_{*}\right) \times \mathcal{P}_{[0, \mathrm{Kl}], M+1}\left(u_{*}\right)
$$

where the second polynomial has constant term equal to 1 and $\tau(f)$ fails the regularity condition (R1.1) is of codimension at least $M+2$ in the space $\overline{\mathcal{P}}$.

Proof We begin by noting that $q_{0}^{\prime}=q_{\mu, 0}+\cdots+q_{m, 0}, w_{0}^{\prime}=w_{0,0}+\cdots+w_{\mathrm{K} 1,0}$ and the equalities $q_{0}^{\prime}=0, w_{0}^{\prime}=1$, expressing the fact that $p$ lies on the vanishing set of $f$ and $g-1$, give two independent conditions for the polynomials $f$ and $g$; we then have remaining $M+1+l$ degrees of freedom for the non-linear defining polynomials $q_{i}^{\prime}$ and $w_{i}^{\prime}$. This immediately gives us non-emptiness of the set $\mathcal{F}$.

For a fixed linear form $L$ in the variables $u_{*}$ we denote by the symbol

$$
\mathcal{P}_{[p ; L]} \subset \mathcal{P}
$$

the affine subspace of pairs $\underline{f}$ such that $q_{0}^{\prime}=0, w_{0}^{\prime}=1$ and $q_{1}^{\prime}=L$.

\subsection{The line connecting the points 0 and $p$}

Let us denote this line by the symbol $[o, p]$. We say we are in the non-special case if $[o, p] \not \subset T_{p} F$, and in the special case if $[o, p] \subset T_{p} F$. First of all we note that in the coordinates $u_{*}$, the line is given by the equations

$$
u_{2}=u_{3}=\cdots=u_{M+1}=0
$$

on the space $T_{p} \mathbb{P}^{*}$. Supposing $[o, p] \not \subset T_{p} F$, we then have

$$
\operatorname{dim}\left\langle\left. u_{2}\right|_{T_{p} F}, \ldots,\left.u_{M+1}\right|_{T_{p} F}\right\rangle=M-1
$$

so that for every $j \geqslant 1$ the space

$$
\left\{\left.q_{j}\right|_{T_{p} F} \mid q_{j} \in \mathcal{P}_{j, M}\right\}
$$

(where $\mathcal{P}_{j, M}$ uses the $M$ variables not equal to $z_{1}$ ) is the whole space of homogeneous polynomials of degree $j$ on $T_{p} F$. Note that it follows from (5) that $q_{\delta}^{\prime}=q_{m, \delta}+(*)$, 
where $(*)$ is a linear combination of terms $u_{1}^{\delta-k} q_{j, k}$ where either $j<m$ or $j=m$ and $k<\delta$.

As the polynomials $q_{j, k}$ are arbitrary of degree $k$ in the variables $u_{2}, \ldots, u_{M+1}$, we conclude that when we consider the pairs $\underline{f}$ in the space $\mathcal{P}_{[p ; L]}$, there is no dependence between any of the polynomials $\left.q_{\delta}^{\prime}\right|_{T_{p} V}$ (and indeed the polynomials $w_{\delta}^{\prime}$ ) where $\delta \geqslant 2$ and so we can use the result on non-singular cyclic covers from [9, Proposition 5.1] (noting that the slight difference in regularity conditions-we go up to $w_{\mathrm{K} l}$ instead of $w_{\mathrm{K} l-1}$ - has no bearing on the final estimate) to get the desired inequality, that the set of pairs $\underline{f}$ in $\mathcal{P}$ which fail the regularity condition (R1.1) has codimension at least $M+1$ in the space $\mathcal{P}[p ; L]$. Since for different linear forms $L \neq L^{\prime}$ the spaces $\mathcal{P}_{[p ; L]}$ and $\mathcal{P}_{\left[p ; L^{\prime}\right]}$ are disjoint, we essentially reduce to the case where the cyclic cover is smooth where the codimension is as required.

Therefore, we assume we are in the special case, that is, $\left.L\right|_{[o, p]} \equiv 0$. Explicitly, this means that the equality

$$
\mu q_{\mu, 0}+\cdots+m q_{m, 0}=0
$$

holds, so we can no longer directly use the previous result. Now note if $\mu \leqslant m-1$ we obtain a new independent condition on $\underline{f}$. We then finish off by using the following proposition.

We set $\mathbb{T}=\mathbb{P}\left(T_{p} F\right) \cong \mathbb{P}^{M-1}$.

Proposition 5.2 Suppose we are in the special case. Then the set of pairs $f \in \mathbb{P}$ such that the system of equations

$$
\left.q_{j}^{\prime}\right|_{\mathbb{T}}=0,\left.\quad w_{k}^{\prime}\right|_{\mathbb{T}}=0, \quad 2 \leqslant j \leqslant m, \quad l \leqslant k \leqslant \mathrm{Kl}-2,
$$

has in $\mathbb{T}$ a positive-dimensional set of solutions, is of codimension at least

$$
\begin{cases}M+1 & \text { if } \mu=m, \\ M & \text { if } \mu \leqslant m-1\end{cases}
$$

in the space $\mathcal{P}[p ; L]$.

Since the regularity conditions would impose that this system would have a zerodimensional set of equations, this inequality is what we require to finish the proof. This is proved in [12, Proposition 3.2], where we are in the case of an arbitrary complete intersection, and unfortunately requires the inequality (1) given in the introduction.

Acknowledgements The author would like to thank Aleksander V. Pukhlikov, who suggested the problem to him and whose support and advice was greatly appreciated, as well as the referee for their various recommendations to improve the paper.

Open Access This article is licensed under a Creative Commons Attribution 4.0 International License, which permits use, sharing, adaptation, distribution and reproduction in any medium or format, as long as you give appropriate credit to the original author(s) and the source, provide a link to the Creative Commons licence, and indicate if changes were made. The images or other third party material in this article are included in the article's Creative Commons licence, unless indicated otherwise in a credit line to the material. If 
material is not included in the article's Creative Commons licence and your intended use is not permitted by statutory regulation or exceeds the permitted use, you will need to obtain permission directly from the copyright holder. To view a copy of this licence, visit http://creativecommons.org/licenses/by/4.0/.

\section{References}

1. Call, F., Lyubeznik, G.: A simple proof of Grothendieck's theorem on the parafactoriality of local rings. In: Heinzer, W.J., et al. (eds.) Commutative Algebra. Contemporary Mathematics, vol. 159, pp. 15-18. American Mathematical Society, Providence (1994)

2. Cheltsov, I.A.: Birationally super-rigid cyclic triple spaces. Izv. Math. 68(6), 1229-1275 (2004)

3. Cheltsov, I.: Double cubics and double quartics. Math. Z. 253(1), 75-86 (2006)

4. Dolgachev, I.: Weighted projective varieties. In: Carrell, J.B. (ed.) Group Actions and Vector Fields. Lecture Notes in Mathematics, vol. 956, pp. 34-71. Springer, Berlin (1982)

5. Iskovskih, V.A., Manin, Ju.I.: Three-dimensional quartics and counterexamples to the Lüroth problem. Mat. Sb. (N.S.) 86(128), 140-166 (1971)

6. Pukhlikov, A.V.: Birationally rigid double Fano hypersurfaces. Sb. Math. 191(5-6), 883-908 (2000)

7. Pukhlikov, A.V.: Birationally rigid Fano iterated double coverings. Izv. Math. 67(3), 555-596 (2003)

8. Pukhlikov, A.V.: Birationally rigid singular Fano hypersurfaces. J. Math. Sci. (N.Y.) 115(3), 2428-2436 (2003)

9. Pukhlikov, A.V.: Birational geometry of algebraic varieties with a pencil of Fano cyclic covers. Pure Appl. Math. Q. 5(2), 641-700 (2009)

10. Pukhlikov, A.: Birationally Rigid Varieties. Mathematical Surveys and Monographs, vol. 190. American Mathematical Society, Providence (2013)

11. Pukhlikov, A.V.: The $4 n^{2}$-inequality for complete intersection singularities. Arnold Math. J. 3(2), 187-196 (2017)

12. Pukhlikov, A.V.: Birationally rigid complete intersections with a singular point of high multiplicity. Proc. Edinburgh Math. Soc. 62(1), 221-239 (2019)

Publisher's Note Springer Nature remains neutral with regard to jurisdictional claims in published maps and institutional affiliations. 